\title{
EXCEPTIONAL MODULES OVER WILD CANONICAL ALGEBRAS
}

\author{
DAWID EDMUND KĘDZIERSKI AND HAGEN MELTZER
}

\begin{abstract}
We show that "almost all" exceptional modules over wild canonical algebra $\Lambda(\underline{p}, \underline{\lambda})$ can be described by matrices having coefficients $\lambda_{i}-\lambda_{j}$, where $\lambda_{i}, \lambda_{j}$ are elements from the parameter sequence $\underline{\lambda}$.

The proof is based on Schofield induction for sheaves in the associated categories of weighted projective lines [15] and an extended version of C. M. Ringel's proof for the ${ }^{\prime \prime} 0,1^{\prime \prime}$ matrix property for exceptional representations for finite acyclic quivers [26].
\end{abstract}

\section{INTRODUCTION}

Canonical algebras were introduced by C. M. Ringel [25]. A canonical algebra $\Lambda$ of quiver type over a field $k$ is a quotient algebra of the path algebra of the quiver $Q$ :

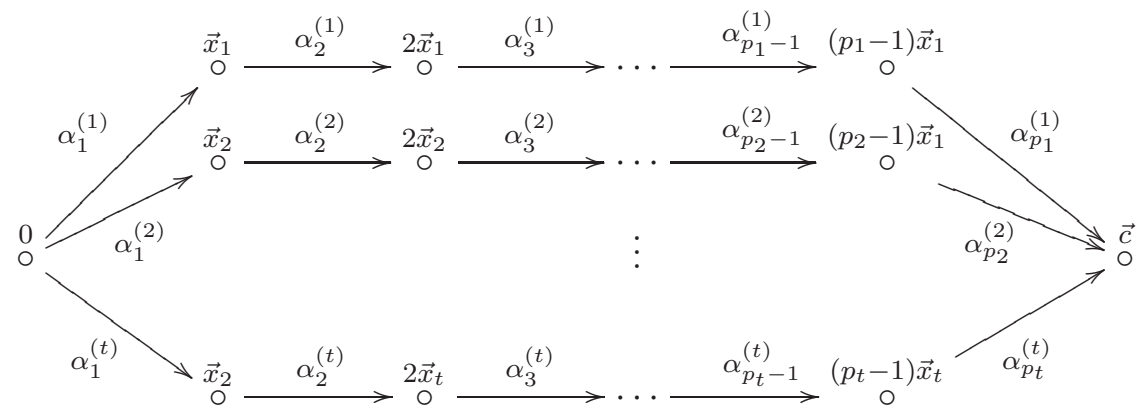

modulo the ideal $I$ defined by the canonical relations

$$
\alpha_{p_{i}}^{(i)} \ldots \alpha_{2}^{(i)} \alpha_{1}^{(i)}=\alpha_{p_{1}}^{(1)} \ldots \alpha_{2}^{(1)} \alpha_{1}^{(1)}+\lambda_{i} \alpha_{p_{2}}^{(2)} \ldots \alpha_{2}^{(2)} \alpha_{1}^{(2)} \quad \text { for } \quad i=3, \ldots, t
$$

where the $\lambda_{i}$ are pairwise distinct non-zero elements of $k$. They are called parameters. The positive integer numbers $p_{i}$ are at least 2 and they are called the weights. Usually we assume that $k$ is algebraically closed, but for many results this is not necessary. The algebra $\Lambda$ depends on a weights sequence $\underline{p}=\left(p_{1}, \ldots, p_{t}\right)$ and a sequence of parameters $\underline{\lambda}=\left(\lambda_{2}, \ldots, \lambda_{t}\right)$. We can assume that $\lambda_{2}=0$ and $\lambda_{3}=1$. We write $\Lambda=\Lambda(\underline{p}, \underline{\lambda})$. Concerning the complexity of the module category over $\Lambda$ there are three types of canonical algebras: domestic, tubular and wild. Recall that the $\Lambda$ is of wild type if and only if the Euler characteristic $\chi_{\Lambda}=(2-t)+\sum_{i=1}^{t} 1 / p_{i}$ is negative.

2010 Mathematics Subject Classification. 16G20, 14F05, 16G60.

Key words and phrases. exceptional module, canonical algebra, wild type, zero-one matrix, Schofield induction, weighted projective line, exceptional pair, generalized Kronecker algebra. 
Denote by $Q_{0}$ the set of vertices and by $Q_{1}$ the set of arrows of the quiver $Q$. Then each finite generated right module over $\Lambda$ is given by finite-dimensional vector spaces $M_{i}$ for each vertex $i$ of $Q_{0}$ and by linear maps $M_{\alpha}: M_{j} \rightarrow M_{i}$ for the arrows $\alpha: i \rightarrow j$ of $Q_{1}$ such that the canonical relations are satisfied. We will usually identify the linear maps with matrices. The category of all this modules we denote by $\bmod (\Lambda)$.

Our aim is to study the possible coefficients, which can appear in the matrices of exceptional modules over wild canonical algebras. In many cases the matrices of special modules can be exhibited by 0,1 matrices. This was shown by $\mathrm{C}$. M. Ringel for exceptional representations of finite acyclic quivers [26] and for indecomposable modules over representation-finite algebras, which is a result of P. Dräxler [10]. In some special case explicit 0, 1 matrices with few nonzero entries have been calculated, so for indecomposable representation of Dynkin quivers by P. Gabriel [1] and indecomposable representation of representation-finite posets by M. Kleiner [16] (see also a result of K. J. Bäckstroem for orders over lattices [1). Among new results we mention a paper of M. Grzecza, S. Kasjan and A. Mróz [13].

The problem of determining matrices for indecomposable modules over canonical algebras has been solved in the case of domestic case. In the case of a field of characteristic different from 2 D. Kussin and the second author computed matrices having entries $0, \pm 1$ for all indecomposable modules, where the entries -1 appears only for very special regular modules [18]. Matrices of indecomposable modules over canonical algebras over an arbitrary field were described in [17. These results were used to determine matrices for exceptional representations for tame quivers [19], [14].

In the case of tubular canonical algebras it was shown in [23] that each exceptional module can be described by matrices having as entries $0, \pm 1$ in the tubular types $(2,3,6),(3,3,3),(2,4,4)$ and for the weight type $(2,2,2,2)$ with a parameter $\lambda$ appear entries $0, \pm 1, \pm \lambda$ and $1-\lambda$. The proof uses universal extensions in the sense of K. Bongartz [3].

Later P. Dowbor, A. Mróz and the second author developed an algorithm and a computer program for explicit calculations of matrices for exceptional modules over tubular canonical algebras [6].

In general little is known about matrices of non-exceptional modules. However in the case of tubular canonical algebra an algorithm for the computation of matrices of non-exceptional modules was developed in [8]. Moreover, explicit formulas for these matrices were obtained in case the module is of integral slope [7. 
Recently the 0,1 property was proved for exceptional objects in the category of nilpotent operators of vector spaces with one invariant subspace, where the nilpotency degree is bounded by 6 [9] and for exceptional objects in the category of nilpotent operators of vector spaces with two incomparable invariant subspaces, where the nilpotency degree is bounded by 3 [4. Both problems are of tubular type and are related to the Birkhoff problem [2] and to recent results on stable vector space categories [20], 21], 22],

The aim of this paper is to present the following result.

Main Theorem. Let $\Lambda=\Lambda(\underline{p}, \underline{\lambda})$ be a wild canonical algebra of quiver type, with $\underline{\lambda}=\left(\lambda_{2}, \cdots, \lambda_{t}\right)$. Then "almost all" exceptional $\Lambda$-modules can be exhibited by matrices involving as coefficients $\lambda_{i}-\lambda_{j}$, where $2 \leq i, j \leq t$.

The notion "almost all" means that in every $\tau_{\mathbb{X}}$-orbit of exceptional modules from a certain place to the right all modules have the expected matrices. We strongly believe that the theorem holds for all exceptional $\Lambda$-modules, but the proof of this fact needs additional arguments.

The theorem will be shown by induction on the rank of a module. Recall, that matrices for modules of rank 0 and 1 are known [18], 23]. Next, by Schofield induction [30] each exceptional $\Lambda$-module $M$ of rank greater than or equal to 2 can be obtained as the central term of a non-split sequence

$$
\text { (*) } 0 \longrightarrow Y^{\oplus v} \longrightarrow M \longrightarrow X^{\oplus u} \longrightarrow 0,
$$

where $(X, Y)$ is an orthogonal exceptional pair in the category $\operatorname{coh}(\mathbb{X})$ of coherent sheaves over the weighted projective line $\mathbb{X}$ corresponding to $\Lambda$ and $(u, v)$ is a dimensional vector of an expceptional representation for the generalized Kronecker algebra having $\operatorname{dim}_{k} \operatorname{Ext}_{\mathbb{X}}^{1}(X, Y)$ arrows [15]. Consequently, like C.M. Ringel in [26] we will study the category $\mathcal{F}(X, Y)$ which consists of all middle term of short exact sequences $(\star)$ for $u, v \in \mathbb{N}_{0}$. This category is equivalent to the module category of generalized Kronecker algebra. Finally, using an alternative description of extension spaces we will assign coefficients for exceptional modules over wild canonical algebras.

The result is part of the $\mathrm{PhD}$ thesis of the first author at Szczecin University in 2017. The authors are thankful to C. M. Ringel for helpful discussion concerning the paper [26].

\section{Notations AND BASIC CONCEPTS}

We recall the concept of a weighted projective line in the sense of GeigleLenzing [12] associated to a canonical algebra $\Lambda=\Lambda(\underline{p}, \underline{\lambda})$. Let $\mathbb{L}=\mathbb{L}(\underline{p})$ be the rank one abelian group with generators $\vec{x}_{1}, \ldots, \vec{x}_{t}$ and relations $p_{1} \vec{x}_{1}=\cdots p_{t} \vec{x}_{t}:=$ 
$\vec{c}$, where $\vec{c}$ is called canonical element. Moreover each element $\vec{y}$ of $\mathbb{L}$ can be written in normal form $\vec{y}=a \vec{c}+\sum_{i=1}^{t} a_{i} \vec{x}_{i}$ with $a \in \mathbb{Z}$ and $0 \leq a_{i}<p_{i}$. The polynomial algebra $k\left[x_{1}, \ldots, x_{t}\right]$ is $\mathbb{L}$-graded, where degree of $x_{i}$ is $\vec{x}_{i}$. Because the polynomials $f_{i}=x_{i}^{p_{i}}-x_{1}^{p_{1}}-\lambda_{i} x_{2}^{p_{2}}$ for $i=3, \ldots t$ are homogeneous, the quotient algebra $S=k\left[x_{1}, \ldots, x_{t}\right] /\left\langle f_{i} \mid i=3, \ldots, t\right\rangle$ is also $\mathbb{L}$-graded. A weighted projective line $\mathbb{X}$ is by definition the projective spectrum of the $\mathbb{L}$-graded algebra $S$. The category of coherent sheaves over $\mathbb{X}$ will be denoted by $\operatorname{coh}(\mathbb{X})$. In other words the category of coherent sheaves $\operatorname{coh}(\mathbb{X})$ is the Serre quotient $\bmod ^{\mathbb{Z}}(S) / \bmod _{0}^{\mathbb{Z}}(S)$, where $\bmod ^{\mathbb{Z}} S$ is the category of finitely generated $\mathbb{Z}$-graded modules over $S$ and $\bmod _{0}^{\mathbb{Z}}(S)$ the subcategory of modules of finite length. It is well known, that each indecomposable sheaf in $\operatorname{coh}(\mathbb{X})$ is a locally free sheaf, called a vector bundle, or a sheaf of finite length. Denote by $\operatorname{vect}(\mathbb{X})\left(\operatorname{resp} \cdot \operatorname{coh}_{0}(\mathbb{X})\right)$ the category of vector bundles (resp. finite length sheaves) on $\mathbb{X}$.

The category $\operatorname{coh}(\mathbb{X})$ is a Hom-finite, abelian $k$-category. Moreover, it is hereditary that means that $\operatorname{Ext}_{\mathbb{X}}^{i}(-,-)=0$ for $i \geq 2$ and it has Serre duality in the form $\operatorname{Ext}_{\mathbb{X}}^{1}(F, G) \cong D \operatorname{Hom}_{\mathbb{X}}\left(G, \tau_{\mathbb{X}} F\right)$, where the Auslander-Reiten translation $\tau_{\mathbb{X}}$ is given by the shift $F \mapsto F(\vec{\omega})$, where $\vec{\omega}:=(t-2) \vec{c}-\sum_{i=1}^{t} \vec{x}_{i}$ denotes the dualizing element, equivalently the category $\operatorname{coh}(\mathbb{X})$ has AuslanderReiten sequences. Moreover, there is a tilting object composed of line bundles with $\operatorname{End}_{\mathbb{X}}(T)=\Lambda$ and it induces an equivalence of a bounded derived category $\mathcal{D}^{b}(\operatorname{coh}(\mathbb{X})) \stackrel{\cong}{\longrightarrow} \mathcal{D}^{b}(\bmod (\Lambda))$.

For coherent sheaves there are well known invariants rank, degree and determinant, which correspond to linear forms rk, deg $: K_{0}(\mathbb{X}) \longrightarrow \mathbb{Z}$ and det $: K_{0}(\mathbb{X}) \longrightarrow$ $\mathbb{L}(\underline{p})$, again called rank, degree and determinant.

Recall that a coherent sheaf $E$ over $\mathbb{X}$ is called exceptional if $\operatorname{Ext}_{\mathbb{X}}^{1}(E, E)=0$ and $\operatorname{End}_{\mathbb{X}}(E)$ is a division ring, in case $k$ is algebraically closed the last means that $\operatorname{End}_{\mathbb{X}}(E)=k$. A pair $(X, Y)$ in $\operatorname{coh}(\mathbb{X})$ is called exceptional if $X$ and $Y$ are exceptional and $\operatorname{Hom}_{\mathbb{X}}(Y, X)=0=\operatorname{Ext}_{\mathbb{X}}^{1}(Y, X)$. Finally, an exceptional pair is orthogonal if additionally $\operatorname{Hom}_{\mathbb{X}}(X, Y)=0$.

The rank of a $\Lambda$-module is defined $\operatorname{rk} M:=\operatorname{dim}_{k} M_{0}-\operatorname{dim}_{k} M_{\vec{c}}$. The rank of a module in this sense equals the rank of the corresponding sheaf in the geometric meaning. We denote by $\bmod _{+}(\Lambda)$ (respectively $\bmod _{-}(\Lambda)$ or $\left.\bmod _{0}(\Lambda)\right)$ the full subcategory consisting of all $\Lambda$-modules, which indecomposable summands of the decomposition into a direct sum have positive (respectively negative or zero) rank. Similarly, by $\operatorname{coh}_{+}(\mathbb{X})$ (resp. $\operatorname{coh}_{-}(\mathbb{X})$ ) we denote the full subcategory of all vector bundles over $\mathbb{X}$, such that the functor $\operatorname{Ext}_{\mathbb{X}}^{1}(T,-)\left(\operatorname{resp}_{\operatorname{Hom}}(T,-)\right)$ vanishes. Under the equivalence $\mathcal{D}^{b}(\operatorname{coh}(\mathbb{X})) \stackrel{\cong}{\longrightarrow} \mathcal{D}^{b}(\bmod (\Lambda))$

- $\operatorname{coh}_{+}(\mathbb{X})$ corresponds to $\bmod _{+}(\Lambda)$ by means of $E \mapsto \operatorname{Hom}_{\mathbb{X}}(T, E)$, 
- $\operatorname{coh}_{0}(\mathbb{X})$ corresponds to $\bmod _{0}(\Lambda)$ by means of $E \mapsto \operatorname{Hom}_{\mathbb{X}}(T, E)$,

- $\operatorname{coh}_{-}(\mathbb{X})[1]$ corresponds to $\bmod _{-}(\Lambda)$ by means of $E[1] \mapsto \operatorname{Ext}_{\mathbb{X}}^{1}(T, E)$, where [1] denotes suspension functor of the triangulated category $D^{b}(\operatorname{coh}(\mathbb{X}))$.

For simplicity we will often identify a sheaf $E$ in $\operatorname{coh}_{+}(\mathbb{X})$ or $\operatorname{coh}_{0}(\mathbb{X})$ with the corresponded $\Lambda$-module $\operatorname{Hom}_{\mathbb{X}}(T, E)$.

\section{EXCEPTIONAL MODULES OF THE SMALL RANK}

First, we start with some matrix notations. For a natural numbers $n$ by $I_{n}$ denote the square diagonal matrix of degree $n$ with each non-zero element equal 1. For a natural number $n$ and $k$ by $X_{n+k, n}$ and $Y_{n+k, n}$ we denote the following matrices.

$X_{n+k, n}:=\left[\begin{array}{ccc} & & \\ & I_{n} & \\ & & \\ \hline 0 & \cdots & 0 \\ \vdots & \ddots & \vdots \\ 0 & \cdots & 0\end{array}\right] \in M_{n+k, n}(k), \quad Y_{n+k, n}:=\left[\begin{array}{ccc}0 & \cdots & 0 \\ \vdots & \ddots & \vdots \\ 0 & \cdots & 0 \\ \hline & & \\ & I_{n} & \end{array}\right] \in M_{n+k, n}(k)$

A $\Lambda$-module of rank zero is called regular. It is well known that the AuslanderReiten quiver of the regular $\Lambda$-modules consists of a family of orthogonal regular tubes with $t$ exceptional tubes $\mathcal{T}_{1}, \ldots, \mathcal{T}_{t}$ of rank $p_{1}, \ldots, p_{t}$, respectively, while the other tubes are homogeneous. Moreover an exceptional regular modules lies in an exceptional tube and its quasi-length is less of the rank of the tube. We will use the description from [18], for the indecomposable regular modules. However we will only quote the shape of the exceptional ones, which lies in the tube $\mathcal{T}_{i}$ for $i \in\{3, \ldots, t\}$. For the tubes $\mathcal{T}_{1}$ and $\mathcal{T}_{2}$ the description is similar. Following the notations from [18] we denote a regular module by $S_{a}^{[l]}$, where $l$ is the quasi-length of $S_{a}^{[l]}$ and $a$ indicates the position on the corresponding floor of the tube. For an exceptional module $S_{a}^{[l]}$ the quasi-length $l<p_{i}$ and so all vector space of $S_{a}^{[l]}$ are zero or one dimensional.

There are 3 cases:

(1) $1 \leq a<p_{i}$ and $0<l<p_{i}-a$,

(2) $1 \leq a<p_{i}$ and $p_{i}-a<l<p_{i}$,

(3) $a=p_{i}$ and $0<l<p_{i}$.

Case (1). 
Then $S_{a}^{[l]}$ has the form



where $0 \longleftarrow k$ and $k \longleftarrow 0$ correspond to the arrow $\alpha_{a}^{(i)}$ and $\alpha_{a+l}^{(i)}$ in the $i-$ th arm.

Case (2).

Let $s:=l-\left(p_{i}-a\right)$. Then $S_{a}^{[l]}$ is the form

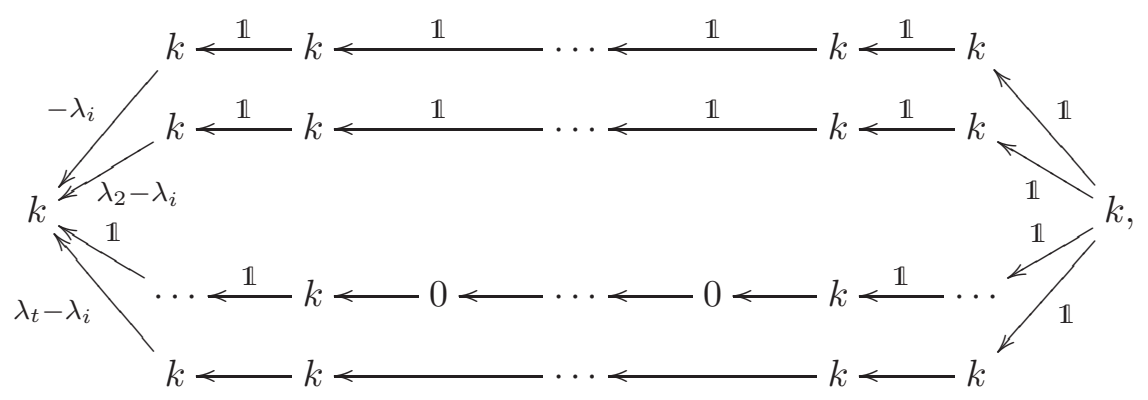

where $k \longleftarrow 0$ and $0 \longleftarrow k$ correspond to the arrow $\alpha_{s}^{(i)}$ and $\alpha_{a}^{(i)}$ in the $i-$ th arm.

Case (3) Then $S_{a}^{[l]}$ is the form

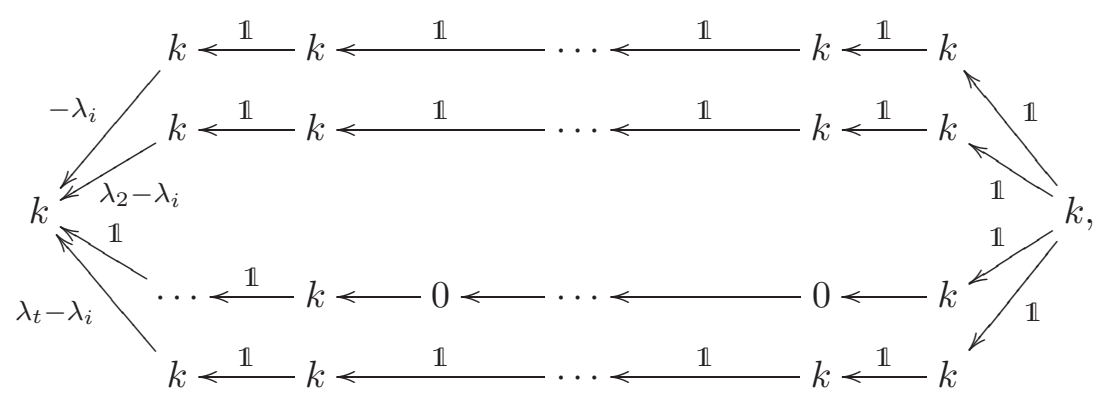

where $k \longleftarrow 0$ and $0 \longleftarrow k$ correspond to the arrow $\alpha_{l}^{(i)}$ and $\alpha_{p_{i}}^{(i)}$ in the $i-$ th arm.

For $\Lambda$-modules of rank one there is the following characterization.

Proposition $3.1([23])$. Let $\Lambda$ be a canonical algebra of quiver type and of arbitrary representation type and $M$ an exceptional $\Lambda$-module of rank 1 . Then $M$ is 
isomorphic to one of the following modules.

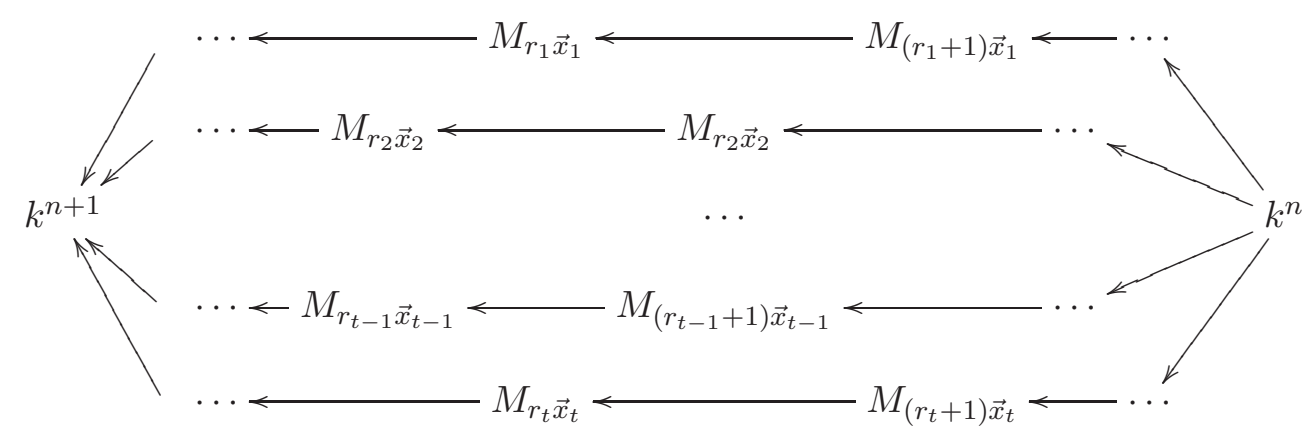

where $r_{i}$ is an integer number such that $0 \leq r_{i}<p_{i}$ for each $i=1,2, \ldots, t$ and

- $M_{s \vec{x}_{i}}=\left\{\begin{array}{cl}k^{n+1} & \text { for } 0 \leq s \leq r_{i} \\ k^{n} & \text { for } r_{i}<s \leq p_{i}\end{array}\right.$

Further the matrices of $M$ are given as follows

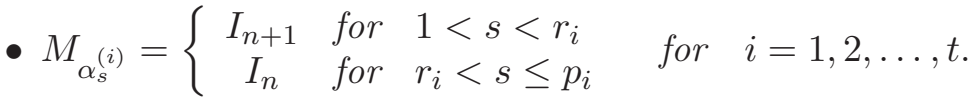

- $M_{\alpha_{r_{1}}^{(1)}}=X_{n+1, n}$

- $M_{\alpha_{r_{2}}^{(2)}}=Y_{n+1, n}$

and for $i=3, \ldots, t$ we distinguish two cases

a) $r_{i}=0$

- $M_{\alpha_{1}^{(i)}}=\left[\begin{array}{cccc}1 & 0 & \cdots & 0 \\ \lambda_{i} & 1 & \cdots & 0 \\ \vdots & \ddots & \ddots & \vdots \\ 0 & 0 & \cdots & 1 \\ 0 & 0 & \cdots & \lambda_{i}\end{array}\right] \in M_{n+1, n}(k)$

b) $r_{i}>0$

- $M_{\alpha_{1}^{(i)}}=\left[\begin{array}{ccccc}1 & 0 & \cdots & 0 & 0 \\ \lambda_{i} & 1 & \cdots & 0 & 0 \\ \vdots & \ddots & \ddots & \vdots & \vdots \\ 0 & 0 & \cdots & 1 & 0 \\ 0 & 0 & \cdots & \lambda_{i} & 1\end{array}\right] \in M_{n+1}(k), \quad \bullet M_{\alpha_{r_{i}}^{(i)}}=X_{n+1, n}$

\section{Schofield induction FRom SheAVES TO MOdUles}

Let $M$ be an exceptional object from $\bmod _{+}(\Lambda)$ of rank greater than or equal to 2 . Then there is a short exact sequence



where $(X, Y)$ is an orthogonal exceptional pair in the category $\operatorname{coh}(\mathbb{X})$, such that the $\operatorname{rk} X<\operatorname{rk} M$ and $\operatorname{rk} Y<\operatorname{rk} M$ and that $(u, v)$ is a dimension vector of an exceptional representation of the generalized Kronecker algebra given by the quiver 
$\Theta(n):$

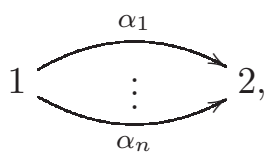

with $n:=\operatorname{dim}_{k} \operatorname{Ext}_{\mathbb{X}}^{1}(X, Y)$ arrows.

This result is called Schofield induction [30] and was applied by C. M. Ringel in the situation of exceptional representations over finite acyclic quivers, hence of hereditary algebras [26].

In the case, that the rank of $X$ or $Y$ is at least 2, we can reapply Schofield induction again and as a result we receive the following sequences

$$
0 \longrightarrow Y_{2}^{\oplus v_{2}} \longrightarrow Y \longrightarrow X_{2}^{\oplus u_{2}} \longrightarrow 0 \quad \text { and } \quad 0 \longrightarrow Y_{3}^{\oplus v_{3}} \longrightarrow X \longrightarrow X_{3}^{\oplus u_{3}} \longrightarrow 0 \text {. }
$$

Because with each successive use of the Schofield induction, the rank of the sheaves decreases, after a finite number of steps we receive pairs of exceptional sheaves of rank 0 or 1 .

This situation is illustrated by the following diagram, which has the shape of a tree like the following one.



Applying the functor $\operatorname{Ext}_{\mathbb{X}}^{1}(T,-)$ to the exact sequence $(\star)$ we see that if $M$ is a $\Lambda$-module, then each sheaf $X_{i_{n}}$ such that there is a path form $M$ to $X_{i_{n}}$ in the tree (1) is also $\Lambda$-module. However, we do not know that a sheave $Y_{*}$ is a $\Lambda$-module.

The following lemma will allows us, by using the $\tau_{\mathbb{X}}$-translation, to shift the tree (1) such that all its components will be $\Lambda$-modules.

Lemma 4.1. Let $\left\{L_{1}, \ldots, L_{m}\right\}$ be a family of line bundles over $\mathbb{X}$. Then there is a natural number $N$ such that $\operatorname{Ext}_{\mathbb{X}}^{1}\left(T, \tau_{\mathbb{X}}^{n} L_{j}\right)=0$ for $j=1, \ldots, m$ and for all $n>N$.

Proof. Let $L_{j}=\mathcal{O}\left(a_{j} \vec{c}+\sum_{i=1}^{t} b_{j, i} \vec{x}_{i}\right)$, where $a_{j} \in \mathbb{Z}, 0 \leq b_{j, i} \leq p_{i}-1$ for $j=$ $1, \ldots, m$ and $i=1, \ldots, t$. We put $N:=\max \left\{\left\lfloor\left(1-a_{j}\right)(t-2)\right\rfloor+1 \mid 1 \leq j \leq m\right\}$. 
Then $\vec{c}+\vec{\omega}-\operatorname{det} \tau_{\mathbb{X}}^{n} L_{j}=\left(1-a_{j}-(n-1)(t-2)\right) \vec{c}+\sum_{i=1}^{t}\left(n-1-b_{j, i}\right) \vec{x}_{i}<0$ for all $n>N$ and $j=1, \ldots, m$. Therefore by Serre duality

$(\triangle) \operatorname{Ext}_{\mathbb{X}}^{1}\left(\mathcal{O}(\vec{c}), \tau_{\mathbb{X}}^{n} L_{j}\right) \cong D \operatorname{Hom}_{\mathbb{X}}\left(\tau_{\mathbb{X}}^{n} L_{j}, \mathcal{O}(\vec{c}+\vec{\omega})\right)=0 \quad$ for $\quad j=1, \ldots, m$.

We have to shown that if $n>N$ then $\operatorname{Ext}_{\mathbb{X}}^{1}\left(\mathcal{O}(\vec{x}), \tau_{\mathbb{X}}^{n} L_{j}\right)=0$ for $0 \leq \vec{x}<\vec{c}$ and $j=1, \ldots, m$. Suppose, that $\operatorname{Ext}_{\mathbb{X}}^{1}\left(\mathcal{O}(\vec{x}), \tau_{\mathbb{X}}^{n} L_{j}\right) \neq 0$ for some $0 \leq \vec{x}<\vec{c}$. Then using Serre duality we get $\operatorname{det} \tau_{\mathbb{X}}^{n} L_{j} \leq \vec{x}+\vec{\omega}$. Because $\vec{x}+\vec{\omega} \leq \vec{c}+\vec{\omega}$, then $\operatorname{det} \tau_{\mathbb{X}}^{n} L_{j} \leq \vec{c}+\vec{\omega}$ and $\operatorname{Ext}_{\mathbb{X}}^{1}\left(\mathcal{O}(\vec{c}), \tau_{\mathbb{X}}^{n} L_{j}\right) \cong \operatorname{DHom}_{\mathbb{X}}\left(\tau_{\mathbb{X}}^{n} L_{j}, \mathcal{O}(\vec{c}+\vec{\omega})\right) \neq 0$ it is contradictory to $(\triangle)$.

Immediately from the lemma above we receive the following corollary.

Corollary 1. There is a natural number $N$ such that for $n>N$ all components of the tree (11) shifted by $\tau_{\mathbb{X}}^{n}$ are $\Lambda$-modules.

Proof. First, note that if the sheaves $X$ and $Y$ in the sequence $(\star)$ are $\Lambda$-modules, then middle term $M$ is a $\Lambda$-module. Next, because there are no nonzero morphisms from finite length sheaves to vector bundles, each finite length sheaf is a $\Lambda$-module.

Let $\mathcal{L}=\left\{L_{i}\right\}_{i \in I}$ be the set of all line bundles appearing in the tree (11). From lemma 4.1 applied to the family $\mathcal{L}$, there is natural number $N$, such that for all natural number $n>N$ the line bundles $\tau_{\mathbb{X}}^{n} L_{i}$ are $\Lambda$-modules for $i \in I$. So the vector bundles in the penultimate parts of the tree (11) are also $\Lambda$-modules. Moving up from the bottom, we get all sheaves in the $\tau_{\mathbb{X}}^{n}$ image are $\Lambda$-modules.

\section{Description of extension SPACES}

Let $(X, Y)$ be an orthogonal exceptional pair in the category $\operatorname{coh}(\mathbb{X})$, this means that $\operatorname{Hom}_{\Lambda}(X, Y)=0=\operatorname{Hom}_{\Lambda}(Y, X), \operatorname{Ext}_{\Lambda}^{1}(Y, X)=0$ and $\operatorname{Ext}_{\Lambda}^{1}(X, Y)=$ $k^{n}$ is non zero space. Assume further that both sheaves $X$ and $Y$ in the sequence ( $\star$ ) are $\Lambda$-modules.

We consider the category $\mathcal{F}(X, Y)$, consisting of all right $\Lambda$-modules $M$, that appear as the middle term in a short exact sequence

$$
0 \longrightarrow Y^{\oplus v} \longrightarrow M \longrightarrow X^{\oplus u} \longrightarrow 0 \text { for some } \quad v, u \in \mathbb{N}_{0} .
$$

It is well known, that the category $\mathcal{F}(X, Y)$ is abelian and has only two simple objects $X$ and $Y$, where the first one is injective simple and the second one is projective simple [24].

Acting like C. M. Ringel in the situation of modules over a hereditary algebras [26] we show that the problem of classifying the objects in the categories $\mathcal{F}(X, Y)$ 
can be reduced to the classification of the modules over the generalized Kronecker algebra, with $n$ arrows.

To do so let $\eta_{1}, \ldots, \eta_{n}$ be a basis of the vector space $\operatorname{Ext}_{\Lambda}^{1}(X, Y)$. Thus we have short exact sequences

$$
\eta_{i}: \quad 0 \longrightarrow Y \longrightarrow Z_{i} \longrightarrow X \longrightarrow 0 \text { for } i=1,2, \ldots n
$$

From the "pull-back" construction there is commutative diagram



where the upper sequence is a universal extension and $Z$ is an exceptional projective object in $\mathcal{F}(X, Y)$. In addition, the projective module $Y \oplus Z$ is progenerator of $\mathcal{F}(X, Y)$. Therefore the functor $\operatorname{Hom}_{\mathbb{X}}(Y \oplus Z,-)$ induces an equivalence between the category $\mathcal{F}(X, Y)$ and the category of modules over the endomorphism algebra $\operatorname{End}_{\Lambda}(Y \oplus Z)$, which is isomorphic to generalized Kronecker algebra $k \Theta(n)$, where $n:=\operatorname{dim}_{k} \operatorname{Ext}_{\Lambda}^{1}(X, Y)$.

Now, we need a more precise description of the above equivalence. Recall from [26] the following concept of extension space between two quiver representations $X$ and $Y$. Let $C^{0}(X, Y)$ and $C^{1}(X, Y)$ be the vector spaces defined as follows

$$
\begin{aligned}
& C^{0}(X, Y):=\bigoplus_{0 \leq \vec{x} \leq \vec{c}} \operatorname{Hom}_{k}\left(X_{\vec{x}}, Y_{\vec{x}}\right), \\
& C^{1}(X, Y):=\bigoplus_{\alpha: \vec{x} \rightarrow \vec{y}} \operatorname{Hom}_{k}\left(X_{\vec{y}}, Y_{\vec{x}}\right),
\end{aligned}
$$

and let $\delta_{X, Y}: C^{0}(X, Y) \longrightarrow C^{1}(X, Y)$ be the linear map, defined by

$$
\delta_{X, Y}\left(\left[f_{\vec{x}}\right]_{0 \leq \vec{x} \leq \vec{c}}\right)=\left[f_{\vec{y}} X_{\alpha}-Y_{\alpha} f_{\vec{z}}\right]_{\alpha: \vec{y} \rightarrow \vec{z}}
$$

where $\alpha$ passing the set $Q_{1}$.

For a path algebra $k Q$ the map $\delta_{X, Y}: C^{0}(X, Y) \longrightarrow C^{1}(X, Y)$ gives also useful description of the extension space of $k Q$-modules [26]. Indeed, then there is $k$-linear isomorphism

$$
\operatorname{Ext}_{k Q}^{1}(X, Y) \cong C^{1}(X, Y) / \operatorname{Im}\left(\delta_{X, Y}\right) .
$$

For modules over a canonical algebra $\Lambda=\Lambda(\underline{p}, \underline{\lambda})$ we must additionally consider the canonical relations of the algebra $\Lambda$. For this we take the subspace 
$U(X, Y)$ of $C^{1}(X, Y)$ containing all $\left[f_{\alpha}\right]_{\alpha \in Q_{1}}$ satisfying the following equations.

$$
\begin{aligned}
& Y_{\omega_{1, p_{i}-1}^{(i)}} f_{\alpha_{p_{i}}^{(i)}}+Y_{\omega_{1, p_{i}-2}^{(i)}} f_{\alpha_{p_{i}-1}^{(i)}} X_{\alpha_{p_{i}}^{(i)}}+\cdots+Y_{\alpha_{1}^{(i)}} f_{\alpha_{2}^{(i)}} X_{\omega_{3, p_{i}}^{(i)}}+f_{\alpha_{1}^{(i)}} X_{\omega_{2, p_{i}}^{(i)}}= \\
= & Y_{\omega_{1, p_{1}-1}^{(1)}} f_{\alpha_{p_{1}}^{(1)}}+Y_{\omega_{1, p_{1}-2}^{(1)}} f_{\alpha_{p_{1}-1}^{(1)}} X_{\alpha_{p_{1}}^{(1)}}+\cdots+Y_{\alpha_{1}^{(1)}} f_{\alpha_{2}^{(1)}} X_{\omega_{3, p_{1}}^{(1)}}+f_{\alpha_{1}^{(1)}} X_{\omega_{2, p_{1}}^{(1)}}+ \\
+ & \lambda_{i}\left(Y_{\omega_{1, p_{2}-1}^{(2)}} f_{\alpha_{p_{2}}^{(2)}}+Y_{\omega_{1, p_{2}-2}^{(2)}} f_{\alpha_{p_{2}-1}^{(2)}} X_{\alpha_{p_{2}}^{(2)}}+\cdots+Y_{\alpha_{1}^{(2)}} f_{\alpha_{2}^{(2)}} X_{\omega_{3, p_{2}}^{(2)}}+f_{\alpha_{1}^{(2)}} X_{\omega_{2, p_{2}}^{(2)}}\right) \\
& \text { for } \quad i=3,4, \ldots, t .
\end{aligned}
$$

Lemma 5.1 (23]). $\operatorname{Ext}_{\Lambda}^{1}(X, Y) \cong U(X, Y) / \operatorname{Im}\left(\delta_{X, Y}\right)$.

We recall the definition of the isomorphism above. Choosing the bases of the spaces $M_{\vec{x}}$ we can assume, that for each arrow $\alpha: i \longrightarrow j$ corresponding map $M_{\alpha}: M_{j} \longrightarrow M_{i}$ have the shape $\left[\begin{array}{c|c}Y_{\alpha} & \varphi_{\alpha} \\ \hline 0 & X_{\alpha}\end{array}\right]$. Then an isomorphism $\phi: \operatorname{Ext}_{\Lambda}^{1}(X, Y) \longrightarrow U(X, Y) / \operatorname{Im}\left(\delta_{X, Y}\right)$ is given by the formula $M=\left(M_{i}, M_{\alpha}\right) \stackrel{\phi}{\mapsto}$ $\left(\varphi_{\alpha}\right)_{\alpha \in Q_{0}}+\operatorname{Im}\left(\delta_{X, Y}\right)$.

Now, we can describe $\Lambda$-modules contained in $\mathcal{F}(X, Y)$, using the matrices of $X, Y$ and the representation of the quiver $\Theta(n)$, which corresponds to the module $M$. Each module in $\mathcal{F}(X, Y)$ can be identified with an element of the extension space $\operatorname{Ext}_{\Lambda}^{1}\left(X^{\oplus u}, Y^{\oplus v}\right)$. Because $X^{\oplus u}=X \otimes k^{u}$ and $Y^{\oplus v}=Y \otimes k^{v}$, then the space $\operatorname{Ext}_{\Lambda}^{1}\left(X^{\oplus u}, Y^{\oplus v}\right)=\operatorname{Ext}_{\Lambda}^{1}\left(X \otimes k^{u}, Y \otimes k^{v}\right)$ is given by the map $\delta_{X \otimes k^{u}, Y \otimes k^{v}}$, where the tensor product is taken over the field $k$. In this situation the vector space $C^{1}\left(X \otimes k^{u}, Y \otimes k^{v}\right)=C^{1}(X, Y) \otimes \operatorname{Hom}_{k}\left(k^{u}, k^{v}\right)$ and also $U\left(X \otimes k^{u}, Y \otimes k^{v}\right)=$ $U(X, Y) \otimes \operatorname{Hom}_{k}\left(k^{u}, k^{v}\right)$. Therefore, from lemma 5.1 and from the commutativity of the following diagram



we obtain that $\operatorname{Ext}_{\Lambda}^{1}\left(X \otimes k^{u}, Y \otimes k^{v}\right) \cong \operatorname{Ext}_{\Lambda}^{1}(X, Y) \otimes \operatorname{Hom}_{k}\left(k^{u}, k^{v}\right)$.

Let $\phi_{1}, \ldots, \phi_{t}$ be a basic of the space $U(X, Y)$. Then $\phi_{1}+\operatorname{Im}\left(\delta_{X, Y}\right), \ldots, \phi_{n}+$ $\operatorname{Im}\left(\delta_{X, Y}\right)$ form a basis of $\operatorname{Ext}_{\Lambda}^{1}(X, Y)$. Now any element in $\operatorname{Ext}_{\Lambda}^{1}\left(X^{\oplus u}, Y^{\oplus v}\right)$ is given by an expression $\sum_{k=1}^{n}\left(f_{\alpha}^{(k)} \otimes A_{k}\right)$, where $A_{k} \in \operatorname{Hom}_{k}\left(k^{u}, k^{v}\right)$ and $\phi_{k}=$ $\left[f_{\alpha}^{(k)}\right]_{\alpha \in Q_{1}}$. Therefore an exceptional $\Lambda$-module $M$, that appears in the sequence $0 \longrightarrow Y^{\oplus v} \longrightarrow M \longrightarrow X^{\oplus u} \longrightarrow 0$, has the form

$M=\left(Y_{\vec{x}}^{\oplus v} \oplus X_{\vec{x}}^{\oplus u},\left[\begin{array}{c|c}Y_{\alpha}^{\oplus v} & \varphi_{\alpha} \\ \hline 0 & X_{\alpha}^{\oplus u}\end{array}\right]\right)_{0 \leq \vec{x} \leq \vec{c}, \alpha \in Q_{1}}$ where $\varphi_{\alpha}=\sum_{m=1}^{n}\left(f_{\alpha}^{(m)} \otimes A_{m}\right)$ 
for an exceptional $\Theta(n)$-representation



Kronecker algebra. An explicit basis for the subspace $U(X, Y)$ we will construct in the next section.

Now we will focus on exceptional modules over the generalized Kronecker algebra. The exceptional modules in this case are known. They are preprojective or preinjective and can be exhibited by matrices having only 0 and 1 entries [26] For recent results concerning modules over generalized Kronecker algebra we refer to [27], [28], [29] [31].

Lemma 5.2. Let $V=k^{v} \frac{A_{1}}{\frac{\vdots}{A_{n}}} k^{u}$ be an exceptional representation of the quiver $\Theta(n)$ and let $A_{m}=\left[a_{i, j}^{(m)}\right]$ for $m=1, \ldots, n$. Then for each pair of natural numbers $(i, j)$ there is at most one index $m$ such that the coefficient $a_{i, j}^{(m)}$ of the matrix $A_{m}$ is non-zero.

Proof. We will use the description of the extension space to show that if for two matrices $A_{1}$ and $A_{2}$ of an exceptional representation $V$ of $\Theta(n)$ non-zero coefficient appear at the same row and column, then $\operatorname{Ext}_{k \Theta(n)}^{1}(V, V) \neq 0$. Consider the map $\delta=\delta_{V, V}: C^{0}(V, V) \longrightarrow C^{1}(V, V)$, where $C^{0}(V, V)=\operatorname{Hom}_{k}\left(k^{v}, k^{v}\right) \oplus \operatorname{Hom}_{k}\left(k^{u}, k^{u}\right)$ and $C^{1}(V, V)=\bigoplus_{m=1}^{n} \operatorname{Hom}_{k}\left(k^{u}, k^{v}\right)$.

Then for $(f, g) \in C^{0}(V, V)$ we have $\delta(f, g)=\bigoplus_{m=1}^{n}\left(f A_{m}-A_{m} g\right)$. The vector space $C^{0}(V, V)$ has a base of the form $\left(e_{i, j}^{v}, 0\right)$ for $1 \leq i, j \leq v$, and $\left(0, e_{i, j}^{u}\right)$ for $1 \leq i, j \leq u$, where $e_{i, j}^{*}$ is an elementary matrix with one non-zero element (equal 1 ) in the $i \times j$-place. Because $A_{m}=\left[a_{i, j}^{(m)}\right]$ for $m=1, \ldots, n$, then $\operatorname{Im}(\delta)$ is generated by the elements

$$
\begin{gathered}
\delta\left(e_{i, j}^{v}, 0\right)=\bigoplus_{m=1}^{n} e_{i, j}^{v} A_{m}=\bigoplus_{m=1}^{n}\left[\begin{array}{ccccccc}
0 & \cdots & 0 & a_{1, j}^{(m)} & 0 & \cdots & 0 \\
\vdots & & \vdots & \vdots & \vdots & & \vdots \\
0 & \cdots & 0 & a_{v, j}^{(m)} & 0 & \cdots & 0
\end{array}\right] \\
\delta\left(0, e_{i, j}^{u}\right)=\bigoplus_{m=1}^{n} A_{m} e_{i, j}^{u}=\bigoplus_{m=1}^{n}\left[\begin{array}{ccccc}
0 & 0 & \cdots & 0 \\
\vdots & \vdots & & \vdots \\
0 & 0 & \cdots & 0 \\
a_{i, 1}^{(m)} & a_{i, 2}^{(m)} & \cdots & a_{i, u}^{(m)} \\
0 & 0 & \cdots & 0 \\
\vdots & \vdots & & \vdots \\
0 & 0 & \cdots & 0
\end{array}\right] .
\end{gathered}
$$


Without lost of generality we can assume that $a_{1,1}^{(m)} \neq 0$ for $m=1,2$. Then the element $x=e_{1,1} \oplus 0 \oplus \cdots \oplus 0$ belongs to $C^{1}(V, V)$ and $x \notin \operatorname{Im}(\delta)$. Therefore $\operatorname{Ext}_{k \Theta(n)}^{1}(V, V) \cong C^{1}(V, V) / \operatorname{Im}(\delta) \neq 0$.

\section{A construction of a base for $U(X, Y)$.}

Let $\Lambda$ be a canonical algebra of the type $\underline{p}=\left(p_{1}, \ldots, p_{t}\right)$ and with parameters $\underline{\lambda}=\left(\lambda_{2}=0, \lambda_{3}=1, \ldots, \lambda_{t}\right)$. A representation $M=\left(\left\{M_{\vec{x}}\right\}_{\vec{x} \in Q_{0}},\left\{M_{\alpha}\right\}_{\alpha \in Q_{1}}\right)$ of an exceptional $\Lambda$-module with positive rank is called acceptable if it satisfies the following conditions.

C1. The matrices $M_{\alpha_{1}^{(1)}}, M_{\alpha_{1}^{(3)}}, M_{\alpha_{1}^{(4)}}, \ldots, M_{\alpha_{1}^{(t)}}$ have entries of the form $\lambda_{a}-\lambda_{b}$ for same $a, b \geq 2$, only.

C2. All other matrices have only 0 and 1 as their coefficients.

C3. For each path $\omega_{u, v}^{(2)}:(u-1) \vec{x}_{2} \longrightarrow v \vec{x}_{2}$ the entries of the matrix $M_{\omega_{u, v}^{(2)}}$ are equal to 0 or 1 .

C4. For each path $\omega_{1, v}^{(i)}: 0 \longrightarrow v \vec{x}_{i}$, where $i \neq 2$ the entries of the matrix $M_{\omega_{1, v}^{(i)}}$ are of the form $\lambda_{a}-\lambda_{b}$ for same $a, b \geq 2$.

C5. For each path $\omega_{u, v}^{(i)}:(u-1) \vec{x}_{i} \longrightarrow v \vec{x}_{i}$, where $i \neq 2$ and $u \geq 2$ the entries of the matrix $M_{\omega_{u, v}^{(i)}}$ are equal to 0 or 1.

The following lemma [23, Lemma 3.4] is useful.

Lemma 6.1. Let $M$ be an acceptable representation of a module in $\bmod _{+}(\Lambda)$. Then by base change we can assume that

$$
M_{\alpha_{j}^{(i)}}=\left[\begin{array}{ccc} 
& & \\
& I_{n} & \\
& & \\
\hline 0 & \cdots & 0 \\
\vdots & \ddots & \vdots \\
0 & \cdots & 0
\end{array}\right] \text { for } 2 \leq j \leq p_{i}, \quad i=3, \ldots, t .
$$

In addition, the matrices $M_{\alpha_{1}^{(i)}}$ have again entries $\lambda_{a}-\lambda_{b}$ for same $a, b \geq 2$.

For an exceptional pair $(X, Y)$ with acceptable representations of $X$ and $Y$ we will construct a basis of subspace $U(X, Y)$, for which each basis vector has only coefficients of the form $\lambda_{a}-\lambda_{b}$. In the case that the $\operatorname{ranks}$ of $\operatorname{rk} X>0$ and $\operatorname{rk} Y>0$ this was done in [23].

Lemma 6.2. Let $X$ and $Y$ be $\Lambda$-modules in $\bmod _{+}(\Lambda)$, with acceptable representations. Then there is basis $F^{(1)}, \ldots, F^{(d)}$ of the subspace $U(X, Y)$, where $F^{(j)}=\left[f_{\alpha}^{(j)}\right]_{\alpha \in Q_{1}}$ satisfies the following properties:

(i) The entries of the matrix $f_{\alpha_{j}^{(2)}}$ are equal 0 or 1 for $1 \leq j \leq p_{i}$, 
(ii) The entries of the matrix $f_{\alpha_{j}^{(i)}}$ are equal 0, 1 for $2 \leq j \leq p_{i}$ and $i=$ $1,3,4, \ldots, t$.

(iii) The entries of the matrix $f_{\alpha_{1}^{(i)}}$ are equal $\lambda_{a}-\lambda_{b}$ for $i=1,3,4, \ldots, t$.

Note, that in the sequence $(\star)$ of the Schofield induction the $\Lambda$-module $Y$ always have the positive rank, but $X$ can have rank zero. In this situation, we need one more lemma.

Lemma 6.3. Let $Y$ and $X$ be an exceptional $\Lambda$-modules such that $Y \in \bmod _{+}(\Lambda)$ and $X \in \bmod _{0}(\Lambda)$. Assume that $X$ and $Y$ have acceptable representations and $X$ lies in the exceptional tube corresponding to $i$-th arm of the canonical algebra. Then there is a base $F^{(1)}, \ldots, F^{(d)}$ of the subspace $U(X, Y)$, where $F^{(j)}=$ $\left[f_{\alpha}^{(j)}\right]_{\alpha \in Q_{1}}$ satisfies the following properties:

(i) The entries of the matrix $f_{\alpha_{j}^{(2)}}$ are equal 0,1 for $1 \leq j \leq p_{2}$,

(ii) The entries of the matrix $f_{\alpha_{j}^{(i)}}$ are equal 0,1 for $1 \leq j \leq p_{i}$,

(iii) The entries of the matrix $f_{\alpha_{1}^{(m)}}$ are equal $\lambda_{a}-\lambda_{b}$ for $m=1,3,4, \ldots, t \wedge$ $m \neq i$,

(iv) The entries of the matrix $f_{\alpha_{j}^{(m)}}$ are equal 0,1 for $2 \leq j \leq p_{m}$ and $m=$ $3,4, \ldots, t$.

Proof. Because $X$ lies in the exceptional tube corresponding to $i$ - th arm of the canonical algebra, then it has a representation of the form $S_{a}^{[l]}$ from the section 3 , such that

(1) $1 \leq a<p_{i}$ and $0<l<p_{i}-a$,

(2) $1 \leq a<p_{i}$ and $p_{i}-a<l<p_{i}$,

(3) $a=p_{i}$ and $0<l<p_{i}$.

In particular, all vector space of $S_{a}^{[l]}$ are zero or one dimensional.

Case (1). From the shape of $S_{a}^{[l]}$ any element of the subspace $U(X, Y)$ has the form

$$
F=\left[\begin{array}{ccccccccc}
0 & \cdots & 0 & 0 & \cdots & 0 & 0 & \cdots & 0 \\
\vdots & & \vdots & \vdots & & \vdots & \vdots & & \vdots \\
0 & \cdots & 0 & 0 & \cdots & 0 & 0 & \cdots & 0 \\
0 & \cdots & 0 & f_{\alpha_{a}^{(i)}} & \cdots & f_{\alpha_{a+l-1}^{(i)}} & 0 & \cdots & 0 \\
0 & \cdots & 0 & 0 & \cdots & 0 & 0 & \cdots & 0 \\
\vdots & & \vdots & \vdots & & \vdots & \vdots & & \vdots \\
0 & \cdots & 0 & 0 & \cdots & 0 & 0 & \cdots & 0
\end{array}\right] .
$$

In addition, the condition describing the subspace $U(X, Y)$ vanishes.

Now we fix $j$ such that $a \leq j<a+l$. Let denote by $e_{r}$ the matrix unit (the matrix with one coefficient 1 namely the coefficient in the row with index $r$, the remaining coefficients are zero). Then $e_{r}$ is an element in $\operatorname{Hom}_{k}\left(X_{j \vec{x}_{i}}, Y_{(j-1) \vec{x}_{i}}\right)=$ 
$\operatorname{Hom}_{k}\left(k, Y_{(j-1)} \vec{x}_{i}\right)$, where $1 \leq r \leq \operatorname{dim}_{k} Y_{(j-1)} \vec{x}_{i}$ and

$$
F_{j}^{r}=\left[\begin{array}{ccccccc}
0 & \cdots & 0 & 0 & 0 & \cdots & 0 \\
\vdots & & \vdots & \vdots & \vdots & & \vdots \\
0 & \cdots & 0 & 0 & 0 & \cdots & 0 \\
0 & \cdots & 0 & e_{r} & 0 & \cdots & 0 \\
0 & \cdots & 0 & 0 & 0 & \cdots & 0 \\
\vdots & & \vdots & \vdots & \vdots & & \vdots \\
0 & \cdots & 0 & 0 & 0 & \cdots & 0
\end{array}\right] .
$$

belongs to $U(X, Y)$ ( $e_{r}$ lies in $j$-th column). It is easy to check, that $F_{j}^{r}$ for $1 \leq r \leq \operatorname{dim}_{k} Y_{(j-1) \vec{x}_{i}}$ and $a \leq j<a+l$ create a base of the subspace $U(X, Y)$.

Case (1). Any element of the subspace $U(X, Y)$ has the form

$$
F_{j}^{r}=\left[\begin{array}{ccccccccc}
f_{\alpha_{1}^{(1)}} & \cdots & f_{\alpha_{s-1}^{(1)}} & f_{\alpha_{s}^{(1)}} & \cdots & f_{\alpha_{a-1}^{(1)}} & f_{\alpha_{a}^{(1)}} & \cdots & f_{\alpha_{p_{1}}^{(1)}} \\
\vdots & & \vdots & \vdots & \vdots & & \vdots & & \\
f_{\alpha_{1}^{(i-1)}} & \cdots & f_{\alpha_{s-1}^{(i-1)}} & f_{\alpha_{s}^{(i-1)}} & \cdots & f_{\alpha_{a-1}^{(i-1)}} & f_{\alpha_{a}^{(i-1)}} & \cdots & f_{p_{p_{i-1}}} \\
f_{\alpha_{1}^{(i)}} & \cdots & f_{\alpha_{s-1}^{(i)}} & 0 & \cdots & 0 & f_{\alpha_{a}^{(i)}} & \cdots & f_{\alpha_{p_{i}}^{(i)}} \\
f_{\alpha_{1}^{(i+1)}} & \cdots & f_{\alpha_{s-1}^{(i+1)}} & f_{\alpha_{s}^{(i+1)}} & \cdots & f_{\alpha_{a-1}^{(i+1)}} & f_{\alpha_{a}^{(i+1)}} & \cdots & f_{\alpha_{p_{i+1}}^{(1)}} \\
\vdots & & \vdots & \vdots & \vdots & & \vdots & & \\
f_{\alpha_{1}^{(t)}} & \cdots & f_{\alpha_{s-1}^{(t)}} & f_{\alpha_{s}^{(t)}} & \cdots & f_{\alpha_{a-1}^{(t)}} & f_{\alpha_{a}^{(t)}} & \cdots & f_{\alpha_{p_{t}}^{(t)}}
\end{array}\right],
$$

where the condition described $U(X, Y)$ has the following shape.

$$
\begin{aligned}
& Y_{\omega_{1, p_{i}-1}^{(i)}} f_{\alpha_{p_{i}}^{(i)}}+\cdots+Y_{\omega_{1, a-1}^{(i)}} f_{\alpha_{a}^{(i)}} \\
= & Y_{\omega_{1, p_{1}-1}^{(1)}} f_{\alpha_{p_{1}}^{(1)}}+Y_{\omega_{1, p_{1}-2}^{(1)}} f_{\alpha_{p_{1}-1}^{(1)}}+\cdots+Y_{\alpha_{1}^{(1)}} f_{\alpha_{2}^{(1)}}+f_{\alpha_{1}^{(1)}} \\
+ & \lambda_{i}\left(Y_{\omega_{1, p_{2}-1}^{(2)}} f_{\alpha_{p_{2}}^{(2)}}+Y_{\omega_{1, p_{2}-2}^{(2)}} f_{\alpha_{p_{2}-1}^{(2)}}+\cdots+Y_{\alpha_{1}^{(2)}} f_{\alpha_{2}^{(2)}}+f_{\alpha_{1}^{(2)}}\right)
\end{aligned}
$$

and for $j \in\{3,4, \ldots, t\}$ and $j \neq i$ we get

$$
\begin{aligned}
& Y_{\omega_{1, p_{j}-1}^{(j)}} f_{\alpha_{p_{j}}^{(j)}}+Y_{\omega_{1, p_{j}-2}^{(j)}} f_{\alpha_{p_{j}-1}^{(j)}}+\cdots+Y_{\alpha_{1}^{(j)}} f_{\alpha_{2}^{(j)}}+f_{\alpha_{1}^{(j)}} \\
= & Y_{\omega_{1, p_{1}-1}^{(1)}} f_{\alpha_{p_{1}}^{(1)}}+Y_{\omega_{1, p_{1}-2}^{(1)}} f_{\alpha_{p_{1}-1}^{(1)}}+\cdots+Y_{\alpha_{1}^{(1)}} f_{\alpha_{2}^{(1)}}+f_{\alpha_{1}^{(1)}} \\
+ & \lambda_{i}\left(Y_{\omega_{1, p_{2}-1}^{(2)}} f_{\alpha_{p_{2}}^{(2)}}+Y_{\omega_{1, p_{2}-2}^{(2)}} f_{\alpha_{p_{2}-1}^{(2)}}+\cdots+Y_{\alpha_{1}^{(2)}} f_{\alpha_{2}^{(2)}}+f_{\alpha_{1}^{(2)}}\right) .
\end{aligned}
$$

We fix $j$ such that $2 \leq j \leq p_{i}$. Again $e_{r}$ is unit matrix belongs to $\operatorname{Hom}_{k}\left(X_{j \vec{x}_{1}}, Y_{(j-1) \vec{x}_{1}}\right)=$ $\operatorname{Hom}_{k}\left(k, Y_{(j-1) \vec{x}_{1}}\right)$ for $1 \leq r \leq \operatorname{dim}_{k} Y_{(j-1) \vec{x}_{1}}$. Then the element

$$
F_{\alpha_{j}^{(1)}}^{r}=\left[\begin{array}{cccccccc}
-Y_{\omega_{1, j-1}^{(1)}} e_{r} & 0 & \cdots & 0 & e_{r} & 0 & \cdots & 0 \\
0 & 0 & \cdots & 0 & 0 & 0 & \cdots & 0 \\
0 & 0 & \cdots & 0 & 0 & 0 & \cdots & 0 \\
\vdots & \vdots & \ddots & \vdots & \vdots & \vdots & \ddots & \vdots \\
0 & 0 & \cdots & 0 & 0 & 0 & \cdots & 0
\end{array}\right]
$$

(where $e_{r}$ lies in the $j$-th column) belongs to $U(X, Y)$. 
We fix $j$ such that $1 \leq j \leq p_{2}$ and let $e_{r}$ belongs to $\operatorname{Hom}_{k}\left(X_{j \vec{x}_{2}}, Y_{(j-1) \vec{x}_{2}}\right)=$ $\operatorname{Hom}_{k}\left(k, Y_{(j-1) \vec{x}_{2}}\right)$ for $1 \leq r \leq \operatorname{dim}_{k} Y_{(j-1)} \vec{x}_{2}$. Then the element

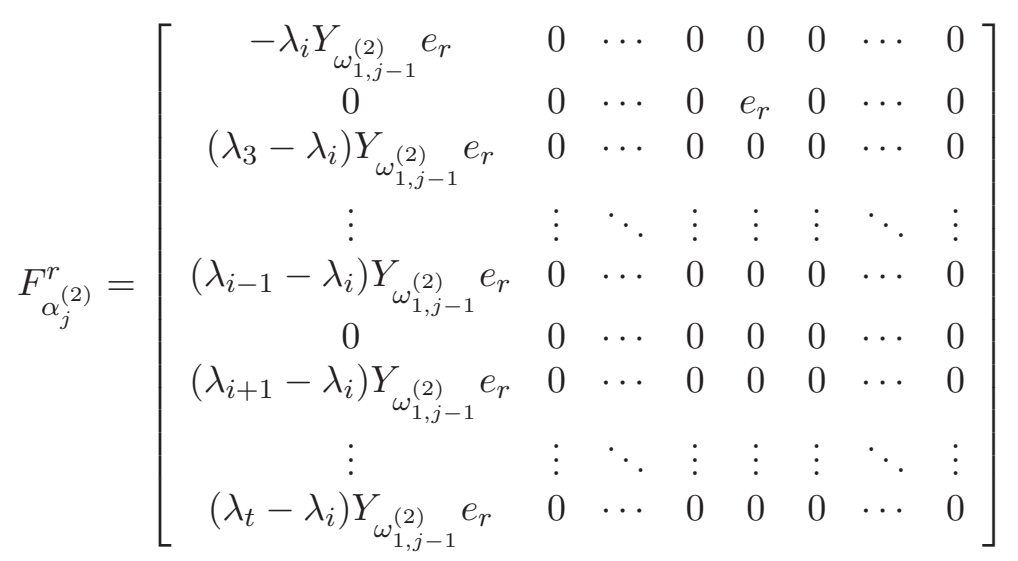

belongs to $U(X, Y)$.

Next, assume that $3 \leq m \leq t, m \neq i$ and $1<j \leq p_{m}$. Let $e_{r}$ be a unit matrix in $\operatorname{Hom}_{k}\left(X_{j \vec{x}_{m}}, Y_{(j-1) \vec{x}_{m}}\right)=\operatorname{Hom}_{k}\left(k, Y_{(j-1) \vec{x}_{m}}\right)$ for $1 \leq r \leq \operatorname{dim}_{k} Y_{(j-1) \vec{x}_{k}}$. Then the element

$$
F_{\alpha_{j}^{r}}^{r}=\left[\begin{array}{cccccccc}
0 & 0 & \cdots & 0 & 0 & 0 & \cdots & 0 \\
0 & 0 & \cdots & 0 & 0 & 0 & \cdots & 0 \\
0 & 0 & \cdots & 0 & 0 & 0 & \cdots & 0 \\
\vdots & \vdots & \ddots & \vdots & \vdots & \vdots & \ddots & \vdots \\
-Y_{\omega_{1, j-1}^{(m)}} e_{r} & 0 & \cdots & 0 & e_{r} & 0 & \cdots & 0 \\
\vdots & \vdots & \ddots & \vdots & \vdots & \vdots & \ddots & \vdots \\
0 & 0 & \cdots & 0 & 0 & 0 & \cdots & 0
\end{array}\right]
$$

belongs to $U(X, Y)$.

Now, we fix $j$ such that $j \in\left\{a, a+1, \ldots, p_{i}\right\}$ and let $e_{r}$ be a unit matrix in $\operatorname{Hom}_{k}\left(X_{j \vec{x}_{i}}, Y_{(j-1) \vec{x}_{i}}\right)=\operatorname{Hom}_{k}\left(k, Y_{(j-1) \vec{x}_{i}}\right)$, where $1 \leq r \leq \operatorname{dim}_{k} Y_{(j-1) \vec{x}_{i}}$. Then the element

$$
F_{\alpha_{j}^{(i)}}^{r}=\left[\begin{array}{ccccccccc}
Y_{\omega_{1, j-1}^{(i)}} e_{r} & 0 & \cdots & 0 & 0 & 0 & \cdots & 0 \\
0 & 0 & \cdots & 0 & 0 & 0 & \cdots & 0 \\
Y_{\omega_{1, j-1}^{(i)}} e_{r} & 0 & \cdots & 0 & 0 & 0 & \cdots & 0 \\
\vdots & & \vdots & \ddots & \vdots & \vdots & \vdots & \ddots & \vdots \\
Y_{\omega_{1, j-1}^{(i)}} e_{r} & 0 & \cdots & 0 & 0 & 0 & \cdots & 0 \\
0 & \cdots & \cdots & 0 & e_{r} & 0 & \cdots & 0 \\
Y_{\omega_{1, j-1}^{(i)}} e_{r} & 0 & \cdots & 0 & 0 & 0 & \cdots & 0 \\
\vdots & \vdots & \ddots & \vdots & \vdots & \vdots & \ddots & \vdots \\
Y_{\omega_{1, j-1}^{(i)}} e_{r} & 0 & \cdots & 0 & 0 & 0 & \cdots & 0
\end{array}\right]
$$

belongs to $U(X, Y)$.

Let $j$ be a natural number such that $j \in\{1,2, \ldots, s-1\}$ and let $e_{r}$ belongs to $\operatorname{Hom}_{k}\left(X_{j \vec{x}_{i}}, Y_{(j-1) \vec{x}_{i}}\right)=\operatorname{Hom}_{k}\left(k, Y_{(j-1)} \vec{x}_{i}\right)$, for $1 \leq r \leq \operatorname{dim}_{k} Y_{(j-1)}$. Then the 
element

$$
F_{\alpha_{j}^{(i)}}^{r}=\left[\begin{array}{ccccccc}
0 & \cdots & 0 & 0 & 0 & \cdots & 0 \\
\vdots & & \vdots & \vdots & \vdots & & \vdots \\
0 & \cdots & 0 & 0 & 0 & \cdots & 0 \\
0 & \cdots & 0 & e_{r} & 0 & \cdots & 0 \\
0 & \cdots & 0 & 0 & 0 & \cdots & 0 \\
\vdots & & \vdots & \vdots & \vdots & & \vdots \\
0 & \cdots & 0 & 0 & 0 & \cdots & 0
\end{array}\right],
$$

belongs to $U(X, Y)$, where $e_{r}$ lies in $j$-th column and $i$-th row.

It is easy to check, that $F_{\alpha}^{r}$ are a base of $U(X, Y)$. In the end, we must check that matrices $f_{\alpha}^{r}$ of basis vectors $F_{\alpha}^{r}$ have desired entries. Because the representation for $Y$ is acceptable, then the matrices $Y_{\omega_{u, v}^{(m)}}$ have only entries $0, \pm 1$, $\pm \lambda_{a}, \lambda_{a}-\lambda_{b}$. Hence the matrix $Y_{\omega_{1, j-1}^{(m)}} e_{r}$ has the same entries. In addition, for $m=2$, the coefficients of matrices $Y_{\omega_{u, v}}^{(2)}$ are equal to 0 or 1 . Therefore matrices $\left(\lambda_{a}-\lambda_{b}\right) Y_{\omega_{1, j-1}^{(2)}} e_{r}$ have only entries $0, \pm 1, \pm \lambda_{a}, \lambda_{a}-\lambda_{b}$.

The case (3) is similar to (2).

Remark, that the coefficients of the form $\lambda_{a}-\lambda_{b}$ occur as coefficients of basis vector of $U(X, Y)$ only if they appear in the acceptable representations $X$ or $Y$. In particular if $X$ and $Y$ are rank modules of rank 1, from Proposition 3.1, then the all basis vectors of $U(X, Y)$ have only coefficients $0, \pm 1$ and $\pm \lambda_{i}$.

\section{Proof OF THE MAIN THEOREM}

Proposition 7.1 (Induction step). Let $M$ be an exceptional module over a canonical algebra $\Lambda$, such that $\operatorname{rk} M \geq 2$. Let $(X, Y)$ be an orthogonal exceptional pair of $\Lambda$-modules, obtained from Schofield induction applied to $M$. If $X$ and $Y$ allows acceptable representations, then also $M$ allows an acceptable representation.

Proof. We will use the basis $F^{(1)}=\left[f_{\alpha}^{(1)}\right]_{\alpha \in Q_{1}}, \ldots, F^{(n)}=\left[f_{\alpha}^{(n)}\right]_{\alpha \in Q_{1}}$ of the subspace $U(X, Y)$ from the lemma 6.2 or lemma 6.3. Because $M$ belong to $\mathcal{F}(X, Y)$, it has the following form.

$M=\left(Y_{\vec{x}}^{\oplus v} \oplus X_{\vec{x}}^{\oplus u},\left[\begin{array}{c|c}Y_{\alpha}^{\oplus v} & \varphi_{\alpha} \\ \hline 0 & X_{\alpha}^{\oplus u}\end{array}\right]\right)_{0 \leq \vec{x} \leq \vec{c}, \alpha \in Q_{1}}$ where $\quad \varphi_{\alpha}=\sum_{m=1}^{n}\left(f_{\alpha}^{(m)} \otimes A_{m}\right)$, for an exceptional $\Theta(n)$-representation $k^{v} \frac{A_{1}}{\vdots} k^{u}$. Recall that all matrices $A_{1}, \ldots, A_{n}$ have entries only 0 and 1 ( see [26]) and moreover from lemma 5.2 non-zero coefficients in consecutive matrices $A_{1}, \ldots, A_{n}$ occur in different places. Therefore the matrix $\sum_{m=1}^{n}\left(f_{\alpha}^{(m)} \otimes A_{m}\right)$ has the same entries as matrices of basis vector $F^{(1)}, \ldots, F^{(n)}$ of $U(X, Y)$. Therefore, because $X$ and $Y$ are acceptable, the 
matrix $M_{\alpha_{1}^{(i)}}$ has entries of the form $\lambda_{a}-\lambda_{b}$ for $i=1,3,4, \ldots, t$ and for $i=2$ only 0 and 1 appear. Next, $M_{\alpha_{j}^{(i)}}$ is a zero-one matrix for $2 \leq j \leq p_{i}$ and $i=1,2, \ldots, t$.

Now, we must check, that for each path $\omega_{l, m}^{(i)}=\alpha_{m}^{(i)} \ldots \alpha_{l}^{(i)}$ the matrix $M_{\omega_{l, m}^{(i)}}=$ $M_{\alpha_{l}^{(i)}} \ldots M_{\alpha_{m}^{(i)}}$ has only expected coefficients. After standard calculations we obtain, that

$$
M_{\omega_{l, m}^{(i)}}=\left[\begin{array}{c|c}
\left(Y_{\omega_{l, m}^{(i)}}\right)^{\oplus v} & \sum Y f X_{\omega_{l, m}^{(i)}} \\
\hline 0 & \left(X_{\omega_{l, m}^{(i)}}\right)^{\oplus u}
\end{array}\right],
$$

where by $\sum Y f X_{\omega_{l, m}^{(i)}}$ we denote

$$
\sum_{j=1}^{n}\left\{Y_{\omega_{l, m-1}^{(i)}} f_{\alpha_{m}^{(i)}}^{(j)}+Y_{\omega_{l, m-2}^{(i)}} f_{\alpha_{m-1}^{(i)}}^{(j)} X_{\alpha_{m}^{(i)}}+\ldots+f_{\alpha_{l}^{(i)}}^{(j)} X_{\omega_{l+1, m}^{(i)}}\right\} \otimes A_{j} .
$$

Because $X$ and $Y$ are acceptable, then $Y_{\omega_{l, m}^{(i)}}$ and $X_{\omega_{l, m}^{(i)}}$ allow only desired entries. Again $\sum Y f X_{\omega_{l, m}^{(i)}}$ has the same coefficients as

$$
Y_{\omega_{l, m-1}^{(i)}} f_{\alpha_{m}^{(i)}}^{(j)}+Y_{\omega_{l, m-2}^{(i)}} f_{\alpha_{m-1}^{(i)}}^{(j)} X_{\alpha_{m}^{(i)}}+\ldots+f_{\alpha_{l}^{(i)}}^{(j)} X_{\omega_{l+1, m}^{(i)}} .
$$

Now the statement concerning the coefficients of the matrices $M_{\omega_{l, m}^{(i)}}$ follows from the explicit description of the elements $F^{(i)}$ by a case by case inspection.

Let us note, that coefficients of the form $\lambda_{a}-\lambda_{b}$ appear only for regular modules. This means that if in the tree (10) there are only vector bundles, then each modules in this tree (after translations) can by established by matrices having coefficients $0, \pm 1, \pm \lambda_{a}$.

Proof of Main Theorem. We prove the fact by induction on the rank of the exceptional module. Remember that a description of exceptional modules of the zero and one rank in section 3, which gives us the start of induction. Let $M$ be an exceptional $\Lambda$-module of rank $\mathrm{r}$ and assume that $r \geq 2$. Then $M$ is corresponds to an exceptional vector bundle over the weighted projective line $\mathbb{X}$ associated to $\Lambda$. By repeated use of Schofield induction, we obtain the figure (1) in the category $\operatorname{coh}(\mathbb{X})$ for $M$. Then from Corollary 1 we can shift all tree, such that each sheaf in the tree is a $\Lambda$-module. Therefore, up to "almost all" we can assume that all tree (1) belongs to the category $\bmod (\Lambda)$. Because all tree components have smaller rank than $M$, then they have acceptable representations. Therefore the claim follows from Proposition 7.1 .

\section{REFERENCES}

1. K. J. Bäckstroem. Orders with infitely many indecomposable latticcs. Phd. Thesis, Göteborg, (1972). 
2. G. Birkhoff, Subgroups of Abelian groups. Proc. Lond. Math. Soc., II. Ser. 38, 385-401, (1934).

3. K. Bongartz Tilted algebras. In Representations of Algebras, Lect. Notes in Math., Vol. 903, 17-32, Springer, Berlin, 17-32, (1981) .

4. P. Dowbor, H. Meltzer. Divectors of indecomposable objecs of nilpotent operatorsof degree 6 with one invariant subspace. Algebr. Repres. Theory, to appear, online https://doi.org/10.1007/s10468-017-9759-z, (2018).

5. P. Dowbor, H. Meltzer. Exceptional objects of nilpotent operators of degree 3 with two incomporable subspaces. Preprint Toruń (2018).

6. P. Dowbor, H. Meltzer, and A. Mróz, An algorithm for the construction of exceptional modules over tubular canonical algebras, J. Algebra 323, no. 10, 2710-2734, (2010).

7. P. Dowbor, H. Meltzer and A. Mróz, Parametrizations for integral slope homogeneous modules over tubular canonical algebras, Algebr. Represent. Theory 17, (1), 321-356, (2014).

8. P. Dowbor, H. Meltzer and A. Mróz, An algorithm for the construction of parametrizing bimodules for homogeneous modules over tubular canonical algebras, Algebr. Represent. Theory 17(1), 357-405, (2014).

9. P. Dowbor, H. Meltzer, M. Schmidmeier The "0,1" property of exceptional objects for nilpotent operators of degree with one invariant subspace. J. Pure Appl. Algebra, to appear.

10. P. Dräxler. Normal forms for representations of representation-finite algebras, J. Symb. Comput. 32, no. 5, 491-497, (2001).

11. P. Gabriel Unzerlegbare Darstellungen I, Manus. Math. 6 , 71-103, (1971).

12. W. Geigle and H. Lenzing, A class of weighted projective curves arising in representation theory of finite dimensional algebras. In: Singularities, representations of algebras, and vector bundles. Springer Lecture Notes in Mathematics 1273 (1987), 265-297, (1987).

13. M. Grzecza, S. Kasjan and A. Mróz, Tree matrices and a matrix reduction algorithm of Belitskii, Fund. Inform. 118, (3) , 253-279, (2012).

14. D. Kędzieski, H. Meltzer. Indecomposable reprentations for extended Dynkin quivers of type $\widetilde{E}_{8}$. Colloqu. Math. 124, no. 1, 95-116, (2011).

15. D.E. Kędzierski and H. Meltzer, Schofield induction for sheaves on weighted projective lines, Commun. Algebra 41, no. 6, 2033-2039, (2013).

16. M. M.Kleiner. Partially ordered sets of finite type. Nauchn. Semin. Leningr. Otd. Mat. Inst. Steklova 28 (1972), 32-41. Engllish translation: J. Sov. Math. 3, 607-615, (1975).

17. S. Komoda and H. Meltzer, Indecomposable modules for domestic canonical algebras in arbitrary characteristic, Int. J. Algebra 2, no. 4, 153-161,(2008).

18. D. Kussin, H. Meltzer. Indecomposable modules for domestic canonical algebras. J. Pure Appl. Algebra 211, no. 2, 471-483, (2007).

19. D. Kussin, H. Meltzer. Indecomposable representations for extended Dynkin quivers. arXiv:math/0612453 (2007).

20. D. Kussin, H. Lenzing, H. Meltzer. Nilpotent operators and weighted projective lines. J. Reine Angew. Math. 685 , 33-71, (2013).

21. D. Kussin, H. Lenzing, H. Meltzer. Triangle singularities, ADE chains and weighted projective lines. Advances in Mathematics 237, 194-251, (2013).

22. D. Kussin, H. Lenzing, H. Meltzer. Nilpotent operators and flags of invariant subspaces. Preprint, Paderborn (2018).

23. H. Meltzer, Exceptional modules for tubular canonical algebras. Algebr. Algebras Represent Theory 10, no. 5, 255-267, (2007).

24. C.M. Ringel, Representations of K-species and bimodules J. Algebra, 41, 269-302, (1976).

25. C.M. Ringel, Tame algebras and integral quadratic forms. Springer Lecture Notes in Mathematics 1099, (1984).

26. C.M. Ringel, Exceptional modules are tree modules. Linear Algebra Appl. 275-276, 471-493, (1998).

27. C.M. Ringel. Indecomposable representations of the Kronecker quivers. Proc. Am. Math. Soc. 141, no. 1, 115-121, (2013).

28. C.M. Ringel. Kronecker mmodules genrated by modules of length 2. Algebras Represent Theory, to appear.

29. C.M. Ringel. The elementary 3-Kronecker modules. Preprint Bielefeld (2016).

30. A. Schofield. Semi-invariants of quivers. J. Lond. Math. Soc., II. Ser. 43, no. 3, 385-395, (1991). 
31. T. Weist Tree modules of the generalised Kronecker quiver. J. Algebra 323, no. 4, 1107-1138, (2010).

Institut of Mathematics, Szczecin University, 70 - 451 Szczecin, Poland

E-mail address: dawid.kedzierski@usz.edu.pl, hagen.meltzer@usz.edu.pl 